\title{
Executive Stock Options as a Screening Mechanism*
}

\author{
Abel Cadenillas ${ }^{\dagger}$ \\ Jakša Cvitanić $\ddagger$ \\ Fernando Zapatero ${ }^{\S}$
}

March 15, 2006

JEL classification: C61, D82, G39

${ }^{*}$ The research of A. Cadenillas was supported by the Social Sciences and Humanities Research Council of Canada. The research of J. Cvitanić was supported in part by NSF grant DMS 04-03575. We are grateful to seminar participants at USC and the University of Pennsylvania and, especially, to Steven Matthews, for comments. Existing errors are our sole responsibility.

${ }^{\dagger}$ Department of Mathematical and Statistical Sciences, University of Alberta, Edmonton, Alberta T6G 2G1, Canada. Ph: (780) 492-0572. Fax: (780) 492-6826. E-mail: acadenil@math.ualberta.ca.

${ }^{\ddagger}$ Caltech, Division of Humanities and Social Sciences, M/C 228-77, 1200 E. California Blvd. Pasadena, CA 91125. Ph: (626) 395-1784. E-mail: cvitanic@hss.caltech.edu

${ }^{\S}$ FBE, Marshall School of Business, USC, Los Angeles, CA 90089-1427. Ph: (213) 740-6538. Fax: (213) 740-6650. E-mail: fzapatero@marshall.usc.edu. 


\title{
Executive Stock Options as a Screening Mechanism
}

\begin{abstract}
We study how and when option grants can be the optimal compensation to screen low-ability executives. In a dynamic setting, we consider the problem of a risk-neutral firm that tries to hire a risk-averse executive whose actions can affect the expected return and volatility of the stock price. Even if the optimal compensation for all types of executives is stock under complete information, it might be optimal to offer options under incomplete information, although a pooling equilibrium in which the optimal contract is stock is also possible. We show that the likelihood of using options increases with the dispersion of types and the size of the firm, and decreases with the availability of growth opportunities for the firm.
\end{abstract}




\section{Introduction}

Stock options are an important component of compensation packages. As reported by Murphy (1999), in 1996, 39\% of the compensation packages of CEO's of companies in the S\&P 500 consisted of options. This percentage goes up to $47 \%$ in 1999. Additionally, $94 \%$ of companies in the S\&P 500 granted options to their CEO's. An obvious explanation for the widespread use of options as compensation has been the accounting advantage that options had not to be expensed the moment they were granted. This is about to change: FASB has decided mandatory expensing of options starting on June 15, 2005. However, it does not seem this will be the end of the use of options as compensation. It is still a relevant question whether options should be part of compensation packages.

Jensen and Murphy (1990) show that the part of the compensation of executives linked to company performance depends mostly on their holdings of stock and options rather than on bonuses (more on this topic can be found in Carpenter 1998, Hall and Leibman 1998, and Murphy 1999). A number of papers consider whether it is optimal to grant options as part of the compensation package. Among the first references in this area are Lambert, Lanen and Larcker (1989) who argue that executive stock options induce a reduction in dividend payments. Yermack (1995) reviews some possible reasons argued in the literature in favor of the use of options for compensation, but finds little empirical support for most of them. Lazear (2001) introduces sorting as a possible argument in favor of option compensation: options will be a cheaper way to compensate optimistic employees. Oyer and Schaefer (2004) find empirical support in favor of sorting. Assef and Santos (2004) argue that option compensation provides the right incentives in a moral hazard setting: in a calibration exercise they find that options can be an optimal contract for plausible risk-aversion parameter values. In a related paper, Palmon, Bar-Yosef, Chen and Venezia (2004) argue in favor of the optimality of granting in-the-money options in a moral hazard setting, versus the standard practice of granting at-the-money options, as examined by Hall and Murphy (2000). Stoughton and Wong (2004) argue against the use of options in industries where firms compete to hire because of the extra flexibility features of options through repricing and resetting policies. Kadan and Swinkels (2004) consider the possibility of bankruptcy: stock is more likely to be the optimal compensation when the probability of bankruptcy is high. One of the potential problems of stock options compensation (see, for instance, John and John, 1993, Johnson and Tian, 2000a, 2000b) is the incentive for the executive to increase volatility, since options values increase with volatility. Carpenter (2000) addresses this problem in a dynamic setting, and shows that this is not necessarily the case for a risk averse executive. Ross (2004) discusses the effects of different compensation schedules and shows that, in fact, convex fees might make a risk averse executive to behave more conservatively.

In this paper we focus on a different motivation for a firm to offer options as compensation. Darrough and Stoughton (1988) show that non-linear compensation 
schemes such as options can provide a better self-selection mechanism than linear schemes. More recently, following a related line of reasoning, Ittner, Lambert and Larcker (2002) find that a major reason for companies to use equity-based compensation is to attract new employees. In their empirical study they do not differentiate between restricted stock and stock options compensation, although they argue that "option-based contracts are (...) more attractive to employees with higher skill levels who have greater ability to take actions that cause their options to finish in the money." Finally, Arya and Mittendorf (2005) show that options provide firms with a tool to screen the true ability of the executive: options will only be accepted by executives who truthfully claim a high ability.

The main contribution of this paper is to provide a framework that is rich enough to study under what conditions options are more likely to be used for screening purposes. We show that option contracts might not be optimal for screening purposes even in a setting of incomplete information: the utility cost resulting from granting options for screening purposes might be too high, and the firm might be better off offering stock and accepting the possibility that all types of executives (even lowtypes) might take the contract.

More explicitly, in our model we consider a risk-neutral firm whose objective is to maximize expected stock price minus cost of the compensation package. The firm needs to hire a risk-averse executive and chooses the number of options and strike price to offer. Executives can affect the dynamics of the stock price in two ways: through the choice of volatility (the manager chooses among a menu of projects) and through costly effort, that affects the expected stock price appreciation. The higher the volatility, the higher also the expected appreciation of the stock price. The riskneutral firm would like the executive to choose a high level of volatility, and apply a high level of effort. There are different executive types, depending on the effect their effort level has on the stock price. In a setting with perfect information about the executive type, the optimal contract might be stock or options, depending on the parameter values of the model. However, as pointed out by Darrough and Stoughton (1988) and Arya and Mittendorf (2005), we show that when the firm is uncertain about the type, it might be optimal (it is not always optimal, however) to offer options, even if the optimal contract with perfect information is stock. Furthermore, we show that options are more likely to be optimal for screening purposes when the dispersion of types is high and when the firm is large, and less likely to be optimal when the growth opportunity of the firm is high.

The paper is structured as follows. In section 2 we describe the economic problem we study in this paper. In section 3 we derive the optimal effort and volatility, as well as the optimal contract. In section 4 we compute some numerical examples and derive the main economic results of the paper. We write the conclusions in section 5 . 


\section{The Model}

The framework in this paper is similar to that of Cadenillas, Cvitanic and Zapatero (2004), but the main difference is that in that paper the firm always knows the type of the executive. In particular, in this paper we consider the problem of a risk-neutral firm that has to decide whether to grant stock or options as compensation to a riskaverse executive that can affect the dynamics of the stock by applying costly effort or choosing the level of volatility. The objective of the firm is to maximize the expected value of the stock price minus the cost of the compensation package. Our results depend on our use of a dynamic setting: we will see that the optimal effort and volatility adapt dynamically to the resulting state.

\subsection{Stock Dynamics}

Our benchmark stock has a price that follows a geometric Brownian motion process,

$$
\frac{d S_{t}}{S_{t}}=\mu d t+\sigma d W_{t}
$$

with starting value $S_{0}$. The process $W$ is a standard Brownian motion process and $\mu$ and $\sigma$ are exogenous constants. However, when the company is managed by the executive whose problem we address in the next subsection, the dynamics of the stock price $S$ is given by

$$
d S_{t}=\delta a_{t} d t+\alpha \sigma_{t} S_{t} d t+\sigma_{t} S_{t} d W_{t}
$$

(without loss of generality, we assume $\mu=0$ ). Here $a$ and $\sigma$ are adapted stochastic processes chosen by the executive, and $\alpha \in(0, \infty)$ is a known constant. In the case of complete information $\delta \in[0, \infty)$ is a known constant, and in the case of incomplete information that we will consider later $\delta$ is a random variable. We assume that $E\left[\int_{0}^{T}\left|a_{t}\right|^{2} d t\right]<\infty$ and $E\left[\int_{0}^{T}\left|\sigma_{t} S_{t}\right|^{2} d t\right]<\infty$. The control $a$ is the level of effort the executive puts in the management of the company. The higher the effort $a$, the higher the expected value of the stock. ${ }^{1}$ Effort produces disutility for the executive in a way we model later. The choice of $\sigma$ is equivalent to the choice of the volatility of the stock: we assume that the executive can choose within a menu of different projects, such that riskier projects also offer higher expected return. We interpret the choice of $\sigma$ as a choice of projects, and the parameter $\alpha$ is a measure of the benefits of taking more risk, and it is a characteristic of the firm. Throughout the paper, we will interpret this parameter as a proxy for growth opportunities available to the firm. The parameter $\delta$ measures the effect the effort of the executive will have on the stock price appreciation. It can be interpreted as an indicator of the type (quality) of the executive, but other interpretations are possible, as we will discuss when we present

\footnotetext{
${ }^{1}$ Baker and Hall (2004) find empirical evidence that indicates that this is a good approximation. We could model an effort whose marginal productivity varies with size, but that do not seem relevant for our purposes of comparing the use of stocks versus options.
} 
the objective of the executive. Carpenter (2000) studies the optimal choice of $\sigma$ for the case in which $\delta=0$.

\subsection{The Executive}

In our model, the executive chooses $a$ and $\sigma$ to maximize expected utility. The executive is risk-averse and effort is costly in terms of utility. The objective of the executive is

$$
e(K, n):=\max _{a, \sigma} E\left[\log \left\{n\left(S_{T}-K\right)^{+}\right\}-\frac{1}{2} \int_{0}^{T} a_{t}^{2} d t\right]
$$

In (2), $n$ is the number of call options or shares of stock the executive receives as part of the compensation package. $T \in(0, \infty)$ is the horizon considered by the executive, that we make equal to the vesting period and the maturity of the option. We ignore the possibility of re-negotiation. As usual, $K \in[0, \infty)$ is the exercise price of the options. The case $K=0$ represents the choice of the firm (whose objective we will discuss below) to grant stock rather than options. The second term of the objective function of the executive represents the disutility from effort and we assume it is quadratic. We point out that instead of characterizing types by the value of their parameter $\delta$ we could have considered a cost parameter in front of the quadratic term of (2). As it will become clear when we present the solution, the two parameterizations are equivalent (there is a one to one mapping that yields the same solution). However, this allows us a more general interpretation of the type: the class of low-type executives will include executives that might be very skilled, but are not really committed to the firm (for personal reasons like family obligations, or for professional reasons such as responsibilities in other firms). The other control, $\sigma$, involves the choice of projects the firm will undertake and has no effect on the disutility of the executive, since it does not require any effort, but it affects the expected value of the compensation package: the executive has a menu of projects and decides the level of risk to undertake. The projects are, in principle, comparable in quality since the projects with higher risk also offer a higher expected return. Higher volatility has two conflicting effects on the executive's utility. On one hand it increases the value of the payoff through higher stock appreciation. On the other hand, it makes the payoff more volatile and, therefore, less desirable from an utility point of view. Options magnify both effects through their implicit leverage properties.

Our choice of logarithmic utility is justified for tractability purposes. A simplifying consequence of our choice is the fact that the number of options $n$ becomes irrelevant for incentive purposes, although it is important to determine the total compensation of the executive and whether the compensation satisfies the participation constraint (that we discuss later). Additionally, we assume that the total compensation package consists of only stock or options. As we will see later, however, the intuition of our results seems to be robust to more general types of utility (at least of the CRRA 
class) and more general compensation packages, at least as long as the equity-based part of the compensation is substantive. In the next section we discuss the solution to this problem.

\subsection{The Firm With Complete Information}

The firm will compensate the executive with stock, or call options on the stock, with maturity $T$. The firm will choose both the number of options $n$ and the strike price $K$. A strike price of 0 indicates that the firm is giving stock to the executive. We assume that the firm is risk-neutral, and cares about the final value of the stock, as well as about the value of the compensation. On the other hand, the firm has to guarantee that the expected utility of the executive is at least as large as a reservation utility $R$. This can be interpreted as the utility that the executive would achieve in the best alternative job offer from another firm. This restriction amounts to a participation constraint, standard in the Principle-Agent literature. Let us define

$$
h(K, n):=\lambda E\left[S_{T}\right]-n E\left[\left(S_{T}-K\right)^{+}\right]
$$

and

$$
A(R):=\left\{(K, n) \in[0, \infty)^{2}: \max _{a, \sigma} E\left[\log \left\{n\left(S_{T}-K\right)^{+}\right\}-\frac{1}{2} \int_{0}^{T} a_{t}^{2} d t\right] \geq R\right\} .
$$

The objective of the firm is

$$
\max _{(K, n) \in A(R)} h(K, n)
$$

where $\lambda$ is an exogenous constant that represents the relative importance for the firm of the expected value of the stock with respect to the compensation package. For a given strike price $K$, the ratio $n / \lambda$ would be an indicator of the "option overhang" (or the proportion of the firm granted in options). The time horizon of the firm matches the time horizon of the executive and the maturity of the options. The value $R$, as we said above, represents the minimum utility the executive has to be able to achieve through the optimal choice of effort and volatility, in order to work for the firm.

\subsection{The Firm With Incomplete Information}

The setting is as before, but we assume further that the firm does not know the type of the executive. That is, the company does not know the value of $\delta$. As we explained above, this is equivalent to the firm not knowing the cost of effort for the executive, which might be a more realistic interpretation in some cases. For example, the executive might have already a reputation (see, for example, Zwiebel 1995 for that type of consideration), but the firm does not really know the level of commitment of the executive. Henceforth, we will assume that there are two types of executives, characterized by the value of $\delta$. More explicitly, the executive can be of high-type $\delta_{H}$ 
with probability $p_{H}$, or of low-type $\delta_{L}$ with probability $p_{L}=1-p_{H}$, with $\delta_{H}>\delta_{L}$. The firm knows the types and their distribution, but cannot tell the particular type of the executive it is negotiating with. We further assume that the types are independent of the Brownian motion process. Additionally, the different executive types have different reservation wages $R_{H}, R_{L}$, with $R_{H}>R_{L}$. As a natural extension of (4), we define $A\left(R_{i}\right), i=H, L$ the set of contracts that satisfies the participation constraint of an executive of type $i$. Finally, we will denote by $E^{i}[X], i=H, L$ the expected value of a random variable $X$ when the executive is of type $i, i=H, L$.

We now define the firm's objective function in several cases, in this setting with two executives with uncertainty, type $H$ with probability $p_{H}$ and type $L$ with probability $p_{L}$.

i) Menu of contracts, no exclusion,

$$
\begin{aligned}
h_{M}\left(K_{H}, K_{L}, n_{H}, n_{L}\right):= & p_{H}\left(\lambda E^{H}\left[S_{T}\right]-n_{H} E^{H}\left[\left(S_{T}-K_{H}\right)^{+}\right]\right) \\
& +p_{L}\left(\lambda E^{L}\left[S_{T}\right]-n_{L} E^{L}\left[\left(S_{T}-K_{L}\right)^{+}\right]\right) .
\end{aligned}
$$

ii) Single contract, no exclusion,

$$
\begin{aligned}
h_{S}(K, n):= & p_{H}\left(\lambda E^{H}\left[S_{T}\right]-n E^{H}\left[\left(S_{T}-K\right)^{+}\right]\right) \\
& +p_{L}\left(\lambda E^{L}\left[S_{T}\right]-n E^{L}\left[\left(S_{T}-K\right)^{+}\right]\right) .
\end{aligned}
$$

iii) Single contract, exclusion of low type,

$$
h_{H}(K, n):=\lambda E^{H}\left[S_{T}\right]-n E^{H}\left[\left(S_{T}-K\right)^{+}\right] .
$$

iv) Single contract, exclusion of high type,

$$
h_{L}(K, n):=\lambda E^{L}\left[S_{T}\right]-n E^{L}\left[\left(S_{T}-K\right)^{+}\right] .
$$

Now denote,

$$
\begin{aligned}
h_{M}^{*}= & \left\{\left(K_{H}, n_{H}\right) \in A\left(R_{H}\right), \notin A\left(R_{L}\right)\right\} \\
& \max _{M}\left(K_{H}, K_{L}, n_{H}, n_{L}\right) \\
h_{S}^{*}= & \max _{\left\{(K, n) \in A\left(R_{H}\right) \cap A\left(R_{L}\right)\right\}} h_{S}(K, n) \\
h_{H}^{*}= & \max _{\left\{(K, n) \in A\left(R_{H}\right), \notin A\left(R_{L}\right)\right\}} h_{H}(K, n) \\
h_{L}^{*}= & \max _{\left\{(K, n) \in A\left(R_{L}\right), \notin A\left(R_{H}\right)\right\}} h_{L}(K, n) .
\end{aligned}
$$


The objective of the firm is to find the pair $\left(K^{*}, n^{*}\right)$, or menu $\left(K_{H}^{*}, K_{L}^{*}, n_{H}^{*}, n_{L}^{*}\right)$, that achieves

$$
\max \left(h_{M}^{*}, h_{S}^{*}, h_{H}^{*}, h_{L}^{*}\right) .
$$

Obviously, a menu of contracts is not necessarily the optimal strategy for the firm. Clearly, that is the case if, for example, the first-best for the high-type is exclusive of the low-type and it is preferable to the first-best for the low-type. But it will also be the case when the cost of excluding the low-type by choosing a second-best contract is lower than the drop in value resulting from a positive probability that a low-type will take the contract.

Finally, we point out that the optimal contract can be a separating or a pooling equilibrium,

i) Separating equilibrium, when the optimal contract is a menu, or it is a single pair $\left(K^{*}, n^{*}\right)$ and $\left(K^{*}, n^{*}\right) \in A\left(R_{H}\right), \notin A\left(R_{L}\right)$ or $\left(K^{*}, n^{*}\right) \in A\left(R_{L}\right), \notin A\left(R_{H}\right)$

ii) Pooling equilibrium, when the optimal contract is a single pair $\left(K^{*}, n^{*}\right)$ and $\left(K^{*}, n^{*}\right) \in A\left(R_{H}\right) \cap A\left(R_{L}\right)$

The solution will be a pooling equilibrium when the first-best of hiring one type of executive (say the high type) is better than the first-best of hiring the other type, and the cost of excluding the second agent (the low type) by choosing a contract outside the acceptable set of the other type (outside $A\left(R_{L}\right)$ ) is higher than the drop in expected value resulting from a positive probability of hiring that type of agent (the low type).

\section{Optimal Strategies}

In this section we derive the solution to the problems of the executive and firm described in the previous section. As we will show, the solution to the problem of the executive is essentially dynamic, since optimal controls are state contingent (unlike in other papers that consider principal-agent models in a dynamic setting).

\subsection{Optimal Effort and Volatility of the Executive}

This problem is similar to the problem considered in Cadenillas, Cvitanić and Zapatero (2004). We repeat the results for convenience. We denote the optimal effort by $\hat{a}$, and the optimal choice of volatility by $\hat{\sigma}$. First we introduce the following auxiliary exponential martingale $Z$,

$$
Z_{t}=\exp \left\{-\frac{1}{2} \alpha^{2} t-\alpha W_{t}\right\}
$$


where $\alpha$ is the parameter in (1) that represents the tradeoff between volatility and expected return of the projects the executive can choose among. Also, consider the following function of time $\bar{T}$,

$$
\bar{T}_{t}=\frac{e^{\alpha^{2}(T-t)}-1}{\alpha^{2}} .
$$

Using the previous notation and given the following quadratic equation in $z$,

$$
\delta^{2} \bar{T}_{0} z^{2}+\left(S_{0}-K\right) z-1=0,
$$

we denote by $\check{z}$ the positive solution of (12):

$$
\check{z}=\frac{1}{2 \delta^{2} \bar{T}_{0}}\left(\left(K-S_{0}\right)+\sqrt{\left(K-S_{0}\right)^{2}+4 \delta^{2} \bar{T}_{0}}\right) .
$$

We now find the optimal controls of the executive:

Proposition 1 Consider the problem of the executive described in sections 2.1 and 2.2. Assume $\delta>0$. The optimal effort $\hat{a}$ of the executive is

$$
\hat{a}_{t}=\delta \check{z} Z_{t} .
$$

The optimal choice of volatility $\hat{\sigma}$ is given by

$$
\hat{\sigma}_{t} S_{t}=\frac{\alpha}{\check{z} Z_{t}}+\alpha \check{z} \delta^{2} Z_{t} \bar{T}_{t} .
$$

The optimal effort and volatility determine that the price of the stock be given by the equation

$$
S_{t}=\frac{1}{\check{z} Z_{t}}+K-\check{z} \delta^{2} Z_{t} \bar{T}_{t}
$$

The value for the executive is

$$
e=\max _{a, \sigma} E\left[\log \left\{n\left(S_{T}-K\right)^{+}\right\}-\frac{1}{2} \int_{0}^{T} a_{t}^{2} d t\right]=\log (n / \check{z})+\frac{\alpha^{2}}{2} T-\frac{1}{2} \delta^{2} \check{z}^{2} \bar{T}_{0} .
$$

Proof: See the Appendix.

$\diamond$

We observe that the optimal effort and volatility can also be written as functions of the price of the stock. That is,

$$
\hat{a}_{t}=\frac{1}{2 \delta^{2} \bar{T}_{t}}\left(\left(K-S_{t}\right)+\sqrt{\left(K-S_{t}\right)^{2}+4 \delta^{2} \bar{T}_{t}}\right) .
$$

and

$$
\begin{aligned}
\hat{\sigma}_{t} S_{t} & =\frac{\alpha \delta}{\hat{a}_{t}}+\alpha \delta \hat{a}_{t} \bar{T}_{t} \\
& =\frac{2 \alpha \delta^{2} \bar{T}_{t}}{\left(K-S_{t}\right)+\sqrt{\left(K-S_{t}\right)^{2}+4 \delta^{2} \bar{T}_{t}}}+\frac{\alpha}{2}\left[\left(K-S_{t}\right)+\sqrt{\left(K-S_{t}\right)^{2}+4 \delta^{2} \bar{T}_{t}}\right] .
\end{aligned}
$$


With respect to the optimal effort, $\hat{a}$ is increasing in the strike price $K$ : as $K$ goes to infinity, the effort goes to infinity as well. The economic reason for that result is that, for a given initial price of the stock, as we increase the strike price, the delta of the option decreases and the implicit leverage in the call increases; this, of course, provides an incentive to the executive to exercise more effort. Obviously, the nature of the utility function (logarithmic, but this property extends to all utilities with CRRA and risk-aversion equal or larger than logarithmic, since they have infinite slope at zero) is such that the executive, who only receives options (or stock) as compensation, is forced to push the stock so that the option will finish in-the-money. However, the relationship between $\hat{a}$ and the strike price is independent of whether the option is in-the-money or not, and seems to be driven by incentives rather than by the type of utility function. Besides, we note that $\check{z}$ is decreasing in $T$, the time to maturity of the option $\left(\bar{T}_{0}\right.$ is increasing in $T$ and $\check{z}$ is decreasing in $\left.\bar{T}_{0}\right)$. Therefore, the larger the maturity of the option, the lower the effort of the executive. The intuition is clear: a larger $T$ has a similar effect on the executive as a lower strike price.

The effect of $\delta$ (the "type" of executive) depends on whether the option is in-, out-, or at-the-money. When the option is at-the-money, the optimal effort is independent of $\delta$, as we can see by substituting (13) in (14). We can also check that when the option is in-the-money the effort is increasing in $\delta$, and when the option is out-of-the-money the optimal effort decreases with $\delta$. The fact that the relationship changes at-themoney is due to the logarithmic utility function and the absence of cash compensation. However, the intuition of this result is useful to understand the main conclusion of the paper (which does not appear to be driven by this result). CRRA forces the executive to put the option in-the-money: when the option is out-of-the-money, lowtype executives apply more effort in order to push the price of the stock upwards; hightype executives prefer to chose higher volatility, that guarantees a higher expected return; the problem with choosing higher volatility for low-type executives is that they will have to apply even higher effort later on, if the higher volatility leads to lower prices of the stock; since high-type executives are more efficient at affecting the price of the stock, they accept this possibility. When options are in-the-money, the main risk (finishing out-of-the-money) is greatly lowered, and then, the higher the marginal productivity of effort, the higher the effort exercised by the executive.

Since $Z$ is a martingale, the expected value of the effort at any point in time is,

$$
E\left[\hat{a}_{t}\right]=\delta \check{z} .
$$

With respect to the effect of $\alpha$, we note that $\bar{T}_{0}$ is increasing in $\alpha$ and, therefore, $\check{z}$ is decreasing in $\alpha$. Expected effort is, then, decreasing in $\alpha$ (everything else constant): the better the menu of projects the executive can choose among, the lower the expected effort of the executive.

The analysis of the optimal volatility is more complicated. Since $\bar{T}_{T}=0$, the second term of (15) decreases in expected value as we approach maturity, and will tend to be negligible relative to the first term. Therefore, for short maturities, optimal 
volatility will tend to decrease with higher strike price. For maturities long enough, the relation will tend to be the opposite. We also see that the volatility is increasing in the type of executive $\delta$ ( $\check{z}$ is decreasing in $\delta$, and $\check{z} \delta^{2}$ is increasing in $\delta$ ). The economic intuition is the same we presented before: a high-type executive can afford more volatility because if the price of the stock drops fast, the high-type is more effective applying effort in order to counteract the drop in the value. It is straightforward to see that the expected value of the volatility at a future date $t$ is

$$
E\left[\hat{\sigma}_{t} S_{t}\right]=\frac{\alpha}{\check{z}} e^{\alpha^{2} t}+\alpha \check{z} \delta^{2} \bar{T}_{t} .
$$

Since $\bar{T}_{t}$ is increasing in $\alpha$, the expected volatility is increasing in $\alpha$. In other words, the higher the expected return-risk tradeoff, the higher the risk the executive will be willing to undertake, on average.

It is also interesting to study the correlation between optimal effort and optimal volatility. By Ito's lemma, and equation (15), the dynamics of the optimal volatility are

$$
d\left(\hat{\sigma}_{t} S_{t}\right)=(\cdot) d t+\alpha^{2}\left(\frac{1}{\check{z} Z_{t}}-\delta^{2} \check{z} Z_{t} \bar{T}_{t}\right) d W_{t} .
$$

Equation (14) yields

$$
d \hat{a}_{t}=(\cdot) d t-\alpha \hat{a}_{t} d W_{t} .
$$

It is clear that their correlation can be either positive or negative. Considering only the instantaneous correlation and ignoring the drift terms, we see that for a short maturity of the option they tend to be negatively correlated, and increases in the optimal effort will be typically associated with decreases in the optimal level of volatility.

\subsection{Optimal Strike Price and Number of Options}

We assume that the firm has full information about the parameters that characterize the dynamics of the stock, as well as the preferences of the executive. The objective of the firm is given by (5).

From equation (16), we obtain

$$
\begin{aligned}
h(K, n) & =\lambda E\left[S_{T}\right]-n E\left[\left(S_{T}-K\right)^{+}\right] \\
& =\lambda\left(g(K) e^{\alpha^{2} T}+K\right)-n g(K) e^{\alpha^{2} T}
\end{aligned}
$$

where

$$
g(K)=\frac{1}{\check{z}}=\frac{2 \delta^{2} \bar{T}_{0}}{\left(K-S_{0}\right)+\sqrt{\left(K-S_{0}\right)^{2}+4 \delta^{2} \bar{T}_{0}}} .
$$

We see that the optimal $n$ is the smallest $n$ that we can take, namely the smallest $n$ such that the participation constraint of the executive is satisfied. As shown in the Appendix, the participation constraint (4) yields,

$$
\frac{1}{2} \delta^{2} \bar{T}_{0} \check{z}^{2}+\log \check{z}-\log n-\frac{\alpha^{2}}{2} T+R=0 .
$$


From this it follows that the firm will take the value of $n$ equal to

$$
n(K)=f(K) \exp \left\{R-\alpha^{2} T / 2+\delta^{2} \bar{T}_{0} f(K)^{2} / 2\right\} .
$$

Here,

$$
f(K)=\frac{1}{g(K)}=\frac{1}{2 \delta^{2} \bar{T}_{0}}\left(\left(K-S_{0}\right)+\sqrt{\left(K-S_{0}\right)^{2}+4 \delta^{2} \bar{T}_{0}}\right) .
$$

Substituting back in $h$, we get the objective function of the firm as the function of one argument only:

$$
\psi(K)=\lambda g(K) e^{\alpha^{2} T}+\lambda K-e^{\alpha^{2} T / 2} \exp \left\{R+\delta^{2} \bar{T}_{0} f(K)^{2} / 2\right\} .
$$

We now state the result about the optimal strike price and the number of options for the firm.

Proposition 2 Consider the firm whose objective is given by (5). The optimal strike price $\hat{K}$ is the value of $K$ that maximizes (26). Then, the optimal number of options is given by $n(\hat{K})$. The value for the firm is $\psi(\hat{K})$.

Proof: See the Appendix.

In the next section we perform some numerical exercises and discuss the economic implications of our model.

\section{Numerical Results and Analysis}

\subsection{The Case of Perfect Information}

It is useful to consider first the shape of the value function of equation (26). In figure 1 we present the value function with perfect information (for a particular set of parameter values) as a function of the strike price $K$ (the number of options/shares $n$ is adjusted to satisfy the participation constraint of the executive, as given by equation (25)). $K=0$ represents stock. We observe that the value function always has the following shape: there is a local maximum for $K=0$, that is, stock, and another local maximum for some high $K$, which represents options. As parameter values change, the relative position of these two points changes, so that sometimes $K=0$ is the global maximum, and sometimes the other point (which also varies in value, as parameters change) is the global maximum. Also, the slope of the value function around $K=0$ is very large in absolute value, so that if $K=0$ is the global maximum, the range of values of $K$ for which the expected value for the firm is larger than for the other local maximum is always small.

To gain some intuition, in table 1 we present results for the case of perfect information. We use the formulas of section 3. In order to find the optimal contract (within the set of contracts considered in our model), we have to find numerically 
the strike price $K$ that maximizes the right-hand side of equation (26). The number of options needed to satisfy the participation constraint of the executive (that is, to clear the reservation wage $R$ ) is given by equation (25). We see that, due to the curvature properties of the value function (see figure 1) the optimal strike price is not continuous in the values of the model parameters. For example, the optimal strike price decreases as $\alpha$ increases and, for some threshold value of $\alpha$ (that will depend on the other parameter values) it jumps to zero, so that the optimal contract consists of stock for that higher value of $\alpha$. In summary, we observe that the strike price (and therefore the delta or implicit leverage of the option) increases with the type of the executive $\delta$ and with $\lambda$ (that we interpret as the size of the firm), and it decreases with the trade-off between risk and volatility, $\alpha$. The intuition is the following: the firm is risk-neutral and would like the executive to be aggressive and choose a high level of volatility, because it offers a high expected return. The higher $\alpha$, the better a high choice of volatility for the firm. Options grants provide executives with a positive incentive (both through higher expected return and through the convexity effect pointed out by Ross, 1973, 2004) and a negative incentive through the increase in risk due to the implicit leverage in options. For executives with high $\delta$ (high type) the positive effect outweighs the risk. The reason is that in bad states executives will have to put more effort to drive up the price of the stock, but for executives with high $\delta$ the cost of that potential extra effort is acceptable. However, for bad executives, the cost of that potential extra effort is too high, and the optimal contract will be options with lower strike price or stock.

\subsection{Incomplete Information: A Particular Case}

We now turn our attention to the case of incomplete information about the type of executive. We focus on a particular case which allows us to derive some interesting conclusions.

As in section 2, we consider only two types of executives, that we call "high" and "low," endowed with different values for $\delta$, that we denote $\delta_{H}$ and $\delta_{L}$, with $\delta_{H}>\delta_{L}$. They might also have different reservation utility values, that we denote, respectively, $R_{H}$ and $R_{L}$. The firm does not know if the parameter that characterizes the type of executive is $\delta_{H}$ or $\delta_{L}$, but it knows these two values and their probabilities. We assume that the firm knows the reservation utility associated with each type: that is, the firm knows that an executive with $\delta_{i}, i \in\{L, H\}$, can command a minimum expected utility $R_{i}, i \in\{L, H\}$.

Darrough and Stoughton (1988) and Arya and Mittendorf (2005) show that option compensation can be optimal for screening purposes. We now want to analyze further that result. To make our analysis simpler, we will focus on cases with the following characteristics:

i) The first-best contract with full information is stock, both for the high and the low-type executives. As discussed in the previous subsection, this is the case 
when $\delta$ is not "too high" and $\alpha$ is "high enough."

ii) We assume that high-type executives have higher reservation value than lowtype executives but, with perfect information, the firm would prefer high-type executives.

iii) We only consider cases in which the optimal contract with perfect information involves more shares for the high-type than for the low-type.

We want to explore if the solution to the problem is such that the firm will optimally offer option grants (instead of stock) due to the agency problem. The numerical solution of this problem is easy to find, given the nature of the value function.

Based on the previous properties, we use the following algorithm to find the optimal contract:

1. First, we find first-best contracts with perfect information for both $H$ and $L$ types, $\left(0, n^{H}\right),\left(0, n^{L}\right){ }^{2}$ In similarity with the notation introduced in section 2 , we denote the value function of the firm for these contracts $h_{H}\left(0, n^{H}\right)$ and $h_{L}\left(0, n^{L}\right)$, respectively. Obviously, since (by choice of parameters) the first-best contract with full information for the executive type $H$ requires more shares than that for the executive type $L, h_{H}\left(0, n^{H}\right)$ is not feasible, because that contract does not exclude the type $L$. However, the single contract $\left(0, n^{H}\right)$ is a candidate for a pooling equilibrium $p_{H} h_{H}\left(0, n^{H}\right)+p_{L} h_{L}\left(0, n^{H}\right)$.

2. Next, we look for a possible menu of contracts. Since (by choice of parameters) $\left(0, n^{H}\right)$ is in the interior of $A\left(R_{L}\right)$, the only possible optimal menu is to offer type $L$ the contract $\left(0, n^{L}\right)$ (which obviously excludes type $H$ ) and find the contract $\left(K^{V}, n^{V}\right) \in A\left(R_{H}\right)$ which excludes type $L$ (it is in the boundary of $\left.A\left(R_{L}\right)\right)$. The value of that contract for the firm is $p_{H} h_{H}\left(K^{V}, n^{V}\right)+p_{L} h_{L}\left(0, n^{L}\right)$.

3. Next, we consider the contract consisting of the other local maximum for executive $H$ (see figure 1 ). Let us denote that contract $\left(K^{M}, n^{M}\right)$. From figure 1, it is clear that very often (given the huge slope in absolute value of the value function at $K=0) h_{H}\left(K^{M}, n^{M}\right)>h_{H}\left(K^{V}, n^{V}\right)$. Therefore, it is possible that $h_{H}\left(K^{M}, n^{M}\right)>p_{H} h_{H}\left(K^{V}, n^{V}\right)+p_{L} h_{L}\left(0, n^{L}\right)$, in which case, $\left(K^{M}, n^{M}\right)$ is a candidate for a separating equilibrium with exclusion of type $L$.

4. Finally, we consider the possible separating equilibrium $\left(0, n^{L}\right)$, which excludes type $H$. Obviously, it is possible that $h_{L}\left(0, n^{L}\right)>h_{H}\left(K^{V}, n^{V}\right)$, in which case this contract would be preferable to the menu.

\footnotetext{
${ }^{2}$ Since we focus on the case in which the optimal contract is stock, we choose parameter values which yield that result. As we showed before, the optimal strike price with perfect information depends monotonically on the values of the parameters of the model, so this is easy to do.
} 
Out of the four contracts considered above, the contract that yields the highest value for the firm will be the equilibrium contract. As we see, the equilibrium can be a no-exclusion menu of contracts, a pooling equilibrium or a separating equilibrium with exclusion of type $H$ or exclusion of type $L$.

In tables 2 and 3 we compute the optimal contract for the case in which the firm does not know the type $(\delta)$ of the executive. In table 2 we consider the case of a "small" firm (measured by the parameter $\lambda$ of the objective function of the firm described in section 2) and in table 3 we consider the case of a "large firm." As we explained before, we focus on cases in which the optimal contract under full information would be stock, both for the "high" and the "low" types, but the firm would prefer the "high" type. We see that, with incomplete information, the optimal contract can be pooling or separating. For our particular examples, when the pooling contract is optimal, the firm offers the first-best contract to type $H$. When the separating contract is optimal, the firm offers options, which are acceptable only to the executives of type $H$. By changing the parameters of the model, we can derive conclusions about the likelihood of the use of options for screening purposes. In particular, we study the likelihood of the use of options for screening purposes as a function of $\delta$, the parameter that measures the ability of the executive, $\alpha$, the parameter that measures the additional expected return resulting from choosing more risk, and $\lambda$, which measures the dilution resulting from granting stock-based compensation and we interpret as a proxy for size of the firm.

It is clear from tables 2 and 3 that the likelihood of the use of options for screening purposes increases with the dispersion of the types. Arguably, the higher the standard deviation of the distribution of types, the more likely is the firm to use options for screening purposes. Higher dispersion of the distribution of types (or, at least, on the priors of the firm with respect to the distribution of types) is likely to happen in more recent industries, with executives with no track record. We have to stress, though, that the parameter $\delta$ can also be interpreted as measuring the degree of commitment of the executive, so the age of the executive is not a clear indicator of high uncertainty about $\delta$.

With respect to $\alpha$, we observe that the likelihood of the use of options decreases with $\alpha$. This parameter is likely to be higher in more recent industries, therefore this effect would go in the opposite direction as the one described in the previous section.

With respect to $\lambda$, we observe that the larger the firm, the more likely it is to use options for screening purposes. Large firms are more likely to be in a mature industry, with lower growth opportunities, which would make this effect consistent with that resulting from $\alpha$.

Finally, we point out that in the examples we have presented the optimal contract is a single contract. However, the optimal contract might be a menu which will typically consist of an option-based contract for type $H$ which satisfies the participation constraint of type $L$, but $L$ is offered instead a stock-based contract that yields the same expected utility for $L$ as the former contract, but with a higher expected value 
for the firm. In our numerical exercises, we could find examples of this sort, but they do not seems as easy to construct as the ones we present in tables 2 and 3 . Additionally, they also involve options as a way to separate the $H$ and the $L$ types.

\section{Conclusions}

We study the use of stock options in compensation packages when the type of the executive is unknown: it has been shown in the literature (for instance, Darrough and Stoughton, 1988, and Arya and Mittendorf, 2005) that a possible role for options is to discourage low-type executives (even if low-type executives are less expensive). We consider a dynamic framework to analyze further when options are more likely to be the optimal contract. In our setting, we can parameterize the dispersion of types, the size of the firm, and the growth opportunity of the firm. We show that options are more likely to be optimal for screening purposes when the dispersion of types is high and when the firm is large, and it is less likely to be optimal when the growth opportunity of the firm is high. 


\section{References}

[1] A. Arya and B. Mittendorf, "Offering Stock Options to Gauge Managerial Talent," Journal of Accounting and Economics 40, 2005, 189-210.

[2] J. Aseff and M. Santos, "Stock Options and Managerial Optimal Contracts," Economic Theory 26, 2005, 813-837.

[3] G. Baker and R. Hall (2004) "CEO Incentives and Firm Size," Journal of Labor Economics 22, 767-798 .

[4] A. Cadenillas, J. Cvitanić and F. Zapatero, "Leverage Decision and Manager Compensation with Choice of Effort and Volatility," Journal of Financial Economics 73 (1) (2004), 71-92.

[5] J. Carpenter, "The Exercise and Valuation of Executive Stock Options," Journal of Financial Economics 48 (2) (1998), 127-158.

[6] J. Carpenter, "Does Option Compensation Increase Managerial Risk Appetite?," Journal of Finance 55 (2000), 2311-2331.

[7] Y.S. Chow and H. Teicher, "Probability Theory: Independence, Interchangeability, Martingales. Second Edition," Springer-Verlag, New York, (1988).

[8] J.C. Cox and C.-F. Huang, "Optimal Consumption and Portfolio Policies when Asset Prices Follow a Diffusion Process," Journal of Economic Theory 49, (1989), 33-83.

[9] M. Darrough and N. Stoughton, "Managerial Compensation: Linear Sharing vs. Bonus Incentive Plans Under Moral Hazard and Adverse Selection," in Economic Analysis of Information and Contracts, G. A. Feltham, A. H. Amershi and W. T. Ziemba, eds., Kluwer Academic Publishers, Boston, (1988).

[10] B. Hall and J. Leibman, "Are CEOs really paid like bureaucrats?," Quarterly Journal of Economics, 113 (3) (1998), 653-691.

[11] B. Hall and K. J. Murphy, "Optimal Exercise Prices for Executive Stock Options," American Economic Review, 2 (2000), 209-214.

[12] C. Ittner, R. Lambert and D. Larcker, "The Structure and Performance Consequences of Equity Grants to Employees of New Economy Firms," working paper, University of Pennsylvania, (2002).

[13] M. Jensen and K. J. Murphy, "Performance Pay and Top-Management Incentives," Journal of Political Economy 98 (1990), 225-264. 
[14] K. John and T. John, "Top Management Compensation and Capital Structure," Journal of Finance 48 (1993), 949-974.

[15] S. Johnson and Y. Tian, "The Value and Incentive Effects of Nontraditional Executive Stock Option Plans," Journal of Financial Economics 57 (2000a), $3-34$.

[16] S. Johnson and Y. Tian, "Indexed Executive Stock Options," Journal of Financial Economics 57 (2000b), 35-64.

[17] O. Kadan and J. Swinkels, "Stock or Options? Managerial Compensation and Bankruptcy - Theory and (Some) Evidence," working paper, Washington University-Saint Louis (2004).

[18] I. Karatzas, J.P. Lehoczky and S.E. Shreve "Optimal Portfolio and Consumption Decisions for a 'Small Investor' on a Finite Horizon," SIAM Journal of Control and Optimization 25 (1987), 1557-1586.

[19] I. Karatzas and S.E. Shreve, "Brownian Motion and Stochastic Calculus," Springer-Verlag, New York, 1991.

[20] R. Lambert, W. Lanen and D. Larcker, "Executive Stock Option Plans and Corporate Dividend Policy," Journal of Financial and Quantitative Analysis 24 (4) (1989), 409-425.

[21] E. Lazear, "Output-Based Pay: Incentives or Sorting?" forthcoming, Research in Labor Economics (2004).

[22] K.J. Murphy, "Executive Compensation," O. Ashenfelter and D. Card, Eds., Handbook of Labor Economics, Vol. III, North Holland, 1999, 2485-2563.

[23] P. Oyer and S. Schaefer, "Why Do Some Firms Give Stock Options To All Employees?: An Empirical Examination of Alternative Theories," forthcoming, Journal of Financial Economics, (2004).

[24] O. Palmon, S. Bar-Yosef, R.-R. Chen and I. Venezia, "Optimal Strike Prices of Stock Options for Effort Averse Executives," working paper, Hebrew University (2004).

[25] S. Ross, "The Economic Theory of Agency: The Principal's Problem," American Economic Review 63 (1973), 134-139.

[26] S. Ross, "Compensation, Incentives, and the Duality of Risk Aversion and Riskiness," Journal of Finance 59 (2004), 207-225.

[27] N. Stoughton and K. Wong, "Option Compensation and Industrial Competition," working paper, UC Irvine (2004). 
[28] D. Yermack, "Do Corporations Award CEO Stock Options Effectively?" Journal of Financial Economics 39 (1995), 237-269.

[29] J. Yong and X.Y. Zhou, "Stochastic Controls: Hamiltonian Systems and HJB Equations," Springer-Verlag, New York, 1999.

[30] J. Zwiebel, "Corporate Conservatism and Relative Compensation," Journal of Political Economy 105 (1995) 1-25. 


\section{A Appendix: Proofs}

\section{A.1 Proof of Proposition 1.}

We consider the more general case in which the executive maximizes

$$
\max _{a, \sigma} E\left[F\left(S_{T}\right)-\int_{0}^{T} G\left(a_{s}\right) d s\right]
$$

where

$$
F(s)=\frac{1}{\gamma}\left[n(s-K)^{+}\right]^{\gamma}, \quad G(a)=\frac{a^{2}}{2}
$$

and $\gamma<1$ is the risk-aversion parameter. The $\log$-utility case $F(x)=\log (x)$ corresponds to $\gamma=0$. We approach this problem by familiar duality/martingale techniques, as introduced by Cox and Huang (1989), Karatzas, Lehoczky and Shreve (1987). Consider the dual function

$$
\tilde{F}(z)=\max _{s \geq 0}[F(s)-s z] .
$$

The maximum is attained at the points of the form

$$
\hat{s}=\hat{s}(z, b)=\left(\left(\frac{z}{n^{\gamma}}\right)^{\frac{1}{\gamma-1}}+K\right) \mathbf{1}_{\left\{\left(\frac{z}{n \gamma}\right)^{\frac{1}{\gamma-1}}>\frac{K \gamma}{1-\gamma}\right\}}+b \mathbf{1}_{\left\{\left(\frac{z}{n^{\gamma}}\right)^{\frac{1}{\gamma-1}}=\frac{K \gamma}{1-\gamma}\right\}},
$$

where $b$ is either 0 or $\left(\frac{z}{n^{\gamma}}\right)^{\frac{1}{\gamma-1}}+K$. Consider also the dual function

$$
\tilde{G}(z)=\max _{a}[-G(a)+\delta a z],
$$

where the maximum is attained at

$$
\hat{a}=\hat{a}(z)=\delta z .
$$

Define the stochastic process

$$
M_{t}=Z_{t} S_{t}-\delta \int_{0}^{t} Z_{s} a_{s} d s
$$

where $Z$ is the exponential martingale defined in (10). Applying Ito's rule, we get

$$
d M_{t}=\left(\sigma_{t}-\alpha\right) S_{t} Z_{t} d W_{t} \quad \text { and } \quad M_{0}=S_{0} .
$$

Obviously, $M$ is a local martingale, but we would like to prove that $M$ is also a martingale. For that purpose, it is good enough to verify the condition

$$
E\left[\sup _{0 \leq t \leq T}\left|M_{t}\right|\right]<\infty
$$


According to the Burkholder-Davis-Gundy inequality (see, for instance, Theorem 3.3.28 of Karatzas and Shreve (1991)), it is enough to check that

$$
E\left[\left(\int_{0}^{T}\left(\sigma_{t}-\alpha\right)^{2} S_{t}^{2} Z_{t}^{2} d t\right)^{1 / 2}\right]<\infty
$$

We observe that, according to Theorem 6.1.6 of Yong and Zhou (1999), $E\left[\sup _{0 \leq t \leq T} Z_{t}^{2}\right]<$ $\infty$. Since $E\left[\int_{0}^{T}\left|\sigma_{t} S_{t}\right|^{2} d t\right]<\infty$, that theorem applied to equation (1) gives $E\left[\sup _{0 \leq t \leq T} S_{t}^{2}\right]<$ $\infty$. Applying Hölder's inequality (see, for instance, Theorem 4.2 of Chow and Teicher (1988)) and again the condition $E\left[\int_{0}^{T}\left|\sigma_{t} S_{t}\right|^{2} d t\right]<\infty$, we note that

$$
\begin{aligned}
E\left[\left(\int_{0}^{T}\left(\sigma_{t} S_{t} Z_{t}\right)^{2} d t\right)^{1 / 2}\right] & \leq E\left[\left(\sup _{0 \leq t \leq T} Z_{t}^{2} \int_{0}^{T}\left(\sigma_{t} S_{t}\right)^{2} d t\right)^{1 / 2}\right] \\
& =E\left[\left(\sup _{0 \leq t \leq T} Z_{t}^{2}\right)^{1 / 2}\left(\int_{0}^{T}\left(\sigma_{t} S_{t}\right)^{2} d t\right)^{1 / 2}\right] \\
& \leq\left(E\left[\sup _{0 \leq t \leq T} Z_{t}^{2}\right]\right)^{1 / 2}\left(E\left[\int_{0}^{T}\left(\sigma_{t} S_{t}\right)^{2} d t\right]\right)^{1 / 2} \\
& <\infty .
\end{aligned}
$$

This implies that

$$
E\left[\left(\int_{0}^{T}\left(\left(\sigma_{t}-\alpha\right) S_{t} Z_{t}\right)^{2} d t\right)^{1 / 2}\right]<\infty
$$

and therefore that $M$ is a martingale. Thus,

$$
E\left[M_{T}\right]=S_{0}
$$

By definitions, we get

$$
E\left[F\left(S_{T}\right)-\int_{0}^{T} G\left(a_{s}\right) d s\right] \leq E\left[\tilde{F}\left(z Z_{T}\right)+\int_{0}^{T} \tilde{G}\left(z Z_{s}\right) d s\right]+z E\left[M_{T}\right]
$$

where we can replace $E\left[M_{T}\right]$ by $S_{0}$. Therefore, the above inequality gives an upper bound for our maximization problem. The upper bound will be attained if the maximums are attained, and if $E\left[M_{T}\right]=S(0)$. In other words, the optimal terminal stock price and the optimal effort $\hat{a}$ are given by

$$
S_{T}=\hat{s}\left(\check{z} Z_{T}, B\right) \quad \text { and } \quad \hat{a}_{t}=\delta \check{z} Z_{t}
$$

where $B$ and $\check{z}$ are chosen so that $B$ is any $\mathcal{F}_{T}$ measurable random variable taking only two possible values, 0 and $\left(\frac{\check{z} Z_{T}}{n^{\gamma}}\right)^{\frac{1}{\gamma-1}}+K$, and so that $E\left[M_{T}\right]=S(0)$. 
For $\gamma=0$, we can choose $B \equiv 0$, and we see that

$$
S_{T}=\frac{1}{\check{z} Z_{T}}+K
$$

Using this and the martingale property of $M$, we get

$$
Z_{t} S_{t}=E\left[\frac{1}{\check{z}}+K Z_{T}-\check{z} \delta^{2} \int_{t}^{T} Z_{s}^{2} d s \mid \mathcal{F}_{t}\right]=\frac{1}{\check{z}}+K Z_{t}-\check{z} \delta^{2} Z_{t}^{2} \bar{T}_{t},
$$

in the notation of (11). In other words,

$$
S_{t}=\frac{1}{\check{z} Z_{t}}+K-\check{z} \delta^{2} Z_{t} \bar{T}_{t}
$$

Using Ito's rule we see that the diffusion term of $S$ is given by

$$
\hat{\sigma}_{t} S_{t}=\frac{\alpha}{\check{z} Z_{t}}+\alpha \check{z} \delta^{2} Z_{t} \bar{T}_{t}
$$

as claimed in (15). We observe that the $\hat{a}$ and $\hat{\sigma}$ defined above are adapted stochastic processes with $E\left[\int_{0}^{T}\left|\hat{a}_{t}\right|^{2} d t\right]<\infty$ and $E\left[\int_{0}^{T}\left|\hat{\sigma}_{t} S_{t}\right|^{2} d t\right]<\infty$. Finally, the requirement $E\left[M_{T}\right]=S_{0}$, obtained by setting $t=0$ in (31), gives

$$
S_{0}=\frac{1}{\check{z}}+K-\check{z} \delta^{2} \bar{T}_{0} .
$$

This is equivalent to (12), and we are done.

\section{A.2 Proof of Proposition 2}

Our first objective is to compute the objective function of the firm

$$
h(K, n):=\lambda E\left[S_{T}\right]-n E\left[\left(S_{T}-K\right)^{+}\right],
$$

and

$$
e=e(n, K):=\max _{a, \sigma} E\left[\log \left\{n\left(S_{T}-K\right)^{+}\right\}-\frac{1}{2} \int_{0}^{T} a_{t}^{2} d t\right] .
$$

It is easily seen that

$$
E\left[Z^{2}(t)\right]=e^{\alpha^{2} t}, \quad E[Z(t)]=1, \quad E\left[Z^{-1}(t)\right]=e^{\alpha^{2} t} .
$$

Thus, according to equation (16),

$$
E\left[S_{t}\right]=\frac{1}{\check{z}} e^{\alpha^{2} t}+K-\check{z} \delta^{2} \bar{T}_{t} .
$$


We also see that $E\left[\left(S_{T}-K\right)^{+}\right]=e^{\alpha^{2} T} / \check{z}$, and, using (13), we verify that the value $h(K, n)$ of $(33)$ is equal to the value $h(K, n)$ of $(22)$.

Finally, using $\hat{a}=\delta \check{z} Z$ and (34), we can compute

$$
e=\log (n / z)+\frac{\alpha^{2}}{2} T-\frac{1}{2} \delta^{2} \check{z}^{2} \bar{T}_{0}
$$

in terms of $z$ and $n$. Here, $\check{z}$ is given in equation (13). We can check that $e(n(K), K)=$ $R$, with $n$ given in (25).

To complete the proof, we note that the firm wishes to maximize the function $h$ as a function of $K$, so that the strike price is non-negative and the executive's rationality constraint is satisfied. 
Table 1

Optimal strike price with perfect information

The column $\hat{K}$ measures the optimal strike price at the initial time for a fixed initial stock price of $S_{0}=100$ and a fixed horizon $T=5 . \hat{K}=0$ means that the optimal contract consists of stock. The column $\hat{n}$ represents the optimal number of call options or shares of stock to offer to the manager as compensation, at the initial time, and for the same initial stock price and time horizon. In this table, $\lambda$ represents the parameter that measures the relative importance of the expected price of the stock with respect to the value of the compensation package, $\alpha$ is the parameter that measures the additional expected return resulting from an additional unit of volatility, $\delta$ is the type of the manager, and $R$ is the reservation compensation of the manager.

\begin{tabular}{|c|c|c|c|c|c|c|c|c|}
\hline & \multicolumn{4}{|c|}{$\alpha=0.1$} & \multicolumn{4}{|c|}{$\alpha=0.2$} \\
\hline & $\delta$ & $R$ & $\hat{K}$ & $\hat{n}$ & $\delta$ & $R$ & $\hat{K}$ & $\hat{n}$ \\
\hline \multirow[t]{9}{*}{$\lambda=100$} & 1 & 1 & 0 & 0.0265 & 1 & 1 & 0 & 0.0246 \\
\hline & 1 & 2 & 0 & 0.072 & 1 & 2 & 0 & 0.0668 \\
\hline & 1 & 5 & 0 & 1.447 & 1 & 5 & 0 & 1.3425 \\
\hline & 2 & 1 & 111.279 & 94.519 & 2 & 1 & 0 & 0.0246 \\
\hline & 2 & 2 & 109.584 & 94.351 & 2 & 2 & 0 & 0.0668 \\
\hline & 2 & 5 & 0 & 1.446 & 2 & 5 & 0 & 1.3414 \\
\hline & 5 & 1 & 131.682 & 94.622 & 5 & 1 & 132.712 & 79.99 \\
\hline & 5 & 2 & 127.862 & 94.508 & 5 & 2 & 128.702 & 79.557 \\
\hline & 5 & 5 & 112.676 & 93.447 & 5 & 5 & 0 & 75.301 \\
\hline \multirow{9}{*}{$\lambda=1000$} & 1 & 1 & 106.758 & 946.793 & 1 & 1 & 0 & 0.0246 \\
\hline & 1 & 2 & 106.825 & 945.912 & 1 & 2 & 0 & 0.0668 \\
\hline & 1 & 5 & 0 & 1.447 & 1 & 5 & 0 & 1.3425 \\
\hline & 2 & 1 & 114.571 & 947.255 & 2 & 1 & 0 & 0.0246 \\
\hline & 2 & 2 & 113.224 & 946.554 & 2 & 2 & 0 & 0.0668 \\
\hline & 2 & 5 & 108.259 & 941.724 & 2 & 5 & 0 & 1.3414 \\
\hline & 5 & 1 & 139.294 & 947.739 & 5 & 1 & 140.673 & 805.674 \\
\hline & 5 & 2 & 136.155 & 947.204 & 5 & 2 & 137.393 & 803.652 \\
\hline & 5 & 5 & 124.934 & 943.952 & 5 & 5 & 125.619 & 791.23 \\
\hline
\end{tabular}


Table 2

Optimal contract with unknown executive type for a "small" firm, that would prefer the high-type with perfect information

The column "Type" indicates whether the executive is of known type "low" (L), known type "high" $(\mathrm{H})$ or unknown type $(\mathrm{U})$, which means the firm thinks it is type $\mathrm{L}$ with probability $50 \%$ and type $\mathrm{H}$ with probability $50 \%$. Columns $\delta^{i}$, which measures the impact of the effort on the expected return, and $R^{i}$, which represents the reservation wage of each executive, characterize the particular type. The column $V^{i}$ records the value of the objective of the firm for that case and optimal contract. The column labeled "equilibrium" denotes whether the resulting equilibrium is "separating" (S) or "pooling" (P). We assume the initial stock price to be $S_{0}=100$ and a fixed horizon $T=5$. $K=0$ means that the optimal contract consists of stock. We also assume that $\lambda$ (the parameter that measures the relative importance of the expected price of the stock with respect to the value of the compensation package) has a value of 1000. $\alpha$ is the parameter that measures the additional expected return resulting from an additional unit of volatility.

\begin{tabular}{|c|c|c|c|c|c|c|c|}
\hline & Type & $\delta^{i}$ & $R^{i}$ & $\hat{K}^{i}$ & $\hat{n}^{i}$ & $V^{i}$ & Equilibrium \\
\hline \multirow[t]{6}{*}{$\alpha=0.25$} & $\mathrm{H}$ & 5 & 2.5 & 0 & 0.103452 & 138646 & \multirow{6}{*}{$\mathrm{P}$} \\
\hline & $\mathrm{L}$ & 4 & 1.5 & 0 & 0.038156 & 137950 & \\
\hline & $\mathrm{U}$ & - & - & 0 & 0.103452 & 138293.5 & \\
\hline & $\mathrm{H}$ & 5 & 2.5 & 0 & 0.103452 & 138646 & \\
\hline & $\mathrm{L}$ & 3 & 1.5 & 0 & 0.038233 & 137397 & \\
\hline & $\mathrm{U}$ & - & - & 136.57 & 707.09 & 138031 & \\
\hline \multirow[t]{6}{*}{$\alpha=0.26$} & $\mathrm{H}$ & 5 & 2.5 & 0 & 0.102131 & 142255 & \multirow{6}{*}{$\mathrm{P}$} \\
\hline & $\mathrm{L}$ & 4 & 1.5 & 0 & 0.037670 & 141531 & \\
\hline & $\mathrm{U}$ & - & - & 0 & 0.102131 & 141888.5 & \\
\hline & $\mathrm{H}$ & 5 & 2.5 & 0 & 0.102131 & 142255 & \\
\hline & $\mathrm{L}$ & 2 & 1.5 & 0 & 0.037803 & 140542 & \\
\hline & $\mathrm{U}$ & - & - & 0 & 0.102131 & 141394 & \\
\hline \multirow[t]{6}{*}{$\alpha=0.249$} & $\mathrm{H}$ & 5 & 2.5 & 0 & 0.103582 & 138298 & \multirow{6}{*}{$\mathrm{S}$} \\
\hline & $\mathrm{L}$ & 4 & 1.5 & 0 & 0.038204 & 137605 & \\
\hline & $\mathrm{U}$ & - & - & 136.55 & 709.212 & 137995 & \\
\hline & $\mathrm{H}$ & 5 & 2.5 & 0 & 0.103582 & 138298 & \\
\hline & $\mathrm{L}$ & 4.5 & 1.5 & 0 & 0.038157 & 137938 & \\
\hline & $\mathrm{U}$ & - & - & 0 & 0.103582 & 138113.5 & \\
\hline
\end{tabular}


Table 3

Optimal contract with unknown executive type for a "large" firm, that would prefer the high-type with perfect information

The column "Type" indicates whether the executive is of known type "low" (L), known type "high" $(\mathrm{H})$ or unknown type $(\mathrm{U})$, which means the firm thinks it is type $\mathrm{L}$ with probability $50 \%$ and type $\mathrm{H}$ with probability $50 \%$. Columns $\delta^{i}$, which measures the impact of the effort on the expected return, and $R^{i}$, which represents the reservation wage of each executive, characterize the particular type. The column $V^{i}$ records the value of the objective of the firm for that case and optimal contract. The column labeled "equilibrium" denotes whether the resulting equilibrium is "separating" (S) or "pooling" (P). We assume the initial stock price to be $S_{0}=100$ and a fixed horizon $T=5$. $K=0$ means that the optimal contract consists of stock. We also assume that $\lambda$ (the parameter that measures the relative importance of the expected price of the stock with respect to the value of the compensation package) has a value of 1100. $\alpha$ is the parameter that measures the additional expected return resulting from an additional unit of volatility.

\begin{tabular}{cccccccc}
\hline & Type & $\delta^{i}$ & $R^{i}$ & $\hat{K}^{i}$ & $\hat{n}^{i}$ & $V^{i}$ & Equilibrium \\
\hline$\alpha=0.25$ & $\mathrm{H}$ & 5 & 2.5 & 0 & 0.103452 & 152513 & \\
& $\mathrm{~L}$ & 4.5 & 1.5 & 0 & 0.0381094 & 152113 & \\
$\mathrm{U}$ & - & - & 0 & 0.103452 & 152308.5 & $\mathrm{P}$ \\
& $\mathrm{H}$ & 5 & 2.5 & 0 & 0.103452 & 152513 & \\
\multirow{5}{*}{$\alpha=0.252$} & $\mathrm{~L}$ & 4 & 1.5 & 0 & 0.038156 & 151746 & \\
& $\mathrm{U}$ & - & - & 136.92 & 778.58 & 152202 & $\mathrm{~S}$ \\
& $\mathrm{H}$ & 5 & 2.5 & 0 & 0.10319 & 153286 & \\
& $\mathrm{~L}$ & 3 & 1.5 & 0 & 0.0381372 & 151900 & \\
& $\mathrm{U}$ & - & - & 0 & 0.10319 & 152588.5 & $\mathrm{P}$ \\
& $\mathrm{H}$ & 5 & 2.5 & 0 & 0.10319 & 153286 & \\
& $\mathrm{~L}$ & 1 & 1.5 & 0 & 0.0382266 & 151192 & \\
& $\mathrm{U}$ & - & - & 136.96 & 769.468 & 152281 & $\mathrm{~S}$ \\
\hline
\end{tabular}




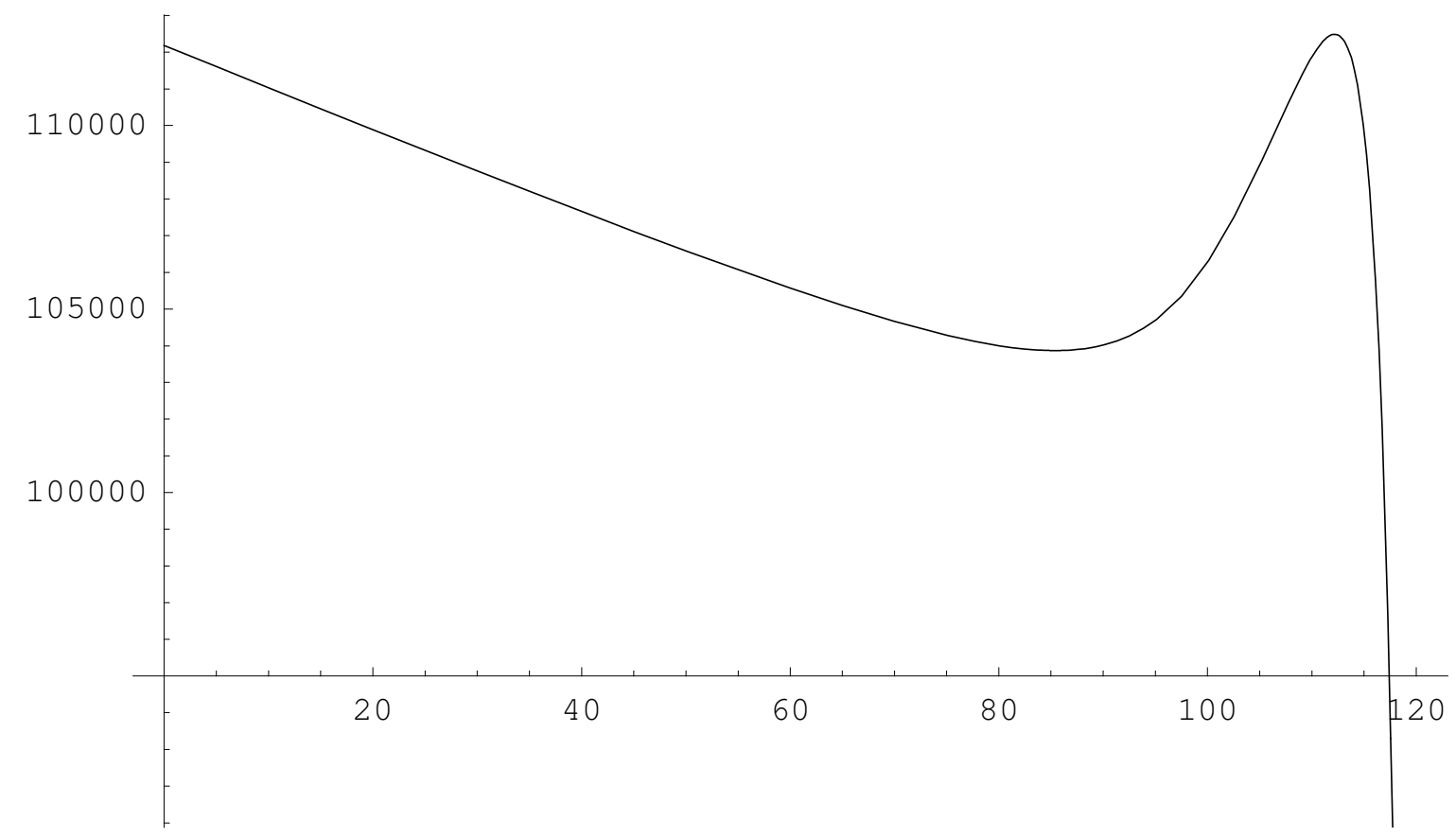

Figure 1: A plot of the value of the objective of the firm as a function of the strike price, for a number of options that satisfies the participation constraint of the executive. Parameter values are $\alpha=0.15, T=5, \lambda=1000, \delta=2.5$, and $R=4.5$. 